\title{
Effects of Various Salts on the Mechanical Properties of Homogeneous Poly(2-hydroxyethyl methacrylate) Hydrogels
}

\author{
Kunio NAKAmURA \\ Department of Polymer Science, Faculty of Science, Hokkaido \\ University, Sapporo 060, Japan.
}

(Received October 27, 1975)

\begin{abstract}
Dynamic mechanical measurements were carried out by a longitudinal vibration method on homogeneous poly(2-hydroxyethyl methacrylate) (PHEMA) hydrogels with added sodium chloride, sodium sulfate, and sodium iodide. Complex tensile moduli were measured at frequencies between 0.006 and $0.6 \mathrm{~Hz}$ and at various temperatures between 0 and $40^{\circ} \mathrm{C}$. The effects of the addition of salts to PHEMA hydrogels on the shape of moduli and compliances and on the monomeric friction coefficient $\zeta$ were studied. It was found that the addition of sodium chloride and sodium sulfate to PHEMA hydrogels causes an increase in $\zeta$ and, that, on the other hand, sodium iodide acts as a plasticizer in the gels. The latter behavior was explained as due to specific ion-polymer interactions. The time-concentration superposition was performed for the system with added salts and the PHEMA gels without added salts, which were studied previously. The concentration reduction which was used in the superposition of the main transition region cannot be applied to the rubber-like region. The values of the reduced compliance in the rubber-like region decrease in the order of $\mathrm{NaI}$ water $>$ water $>\mathrm{NaCl}$-water, $\mathrm{Na}_{2} \mathrm{SO}_{4}$-water. This effect was interpreted in terms of the difference in the influence of the added salts to a diluent-induced structure in the PHEMA gels.
\end{abstract}

KEY WORDS Viscoelasticity / Poly(2-hydroxyethyl methacrylate) / Hydrogel / Monomeric Friction Coefficient / Fujita Theory / BerryFox Theory /

Amphiphilic 2-hydroxyethyl methacrylate polymers (PHEMA) crosslinked by a small amount of ethylene glycol dimethacrylate have been applied in medicine to a still increasing degree, especially as materials for soft contact lenses. ${ }^{1}$ The mechanical behavior of PHEMA in the swollen state prepared in the absence and/or presence of water has been investigated by a number of workers. ${ }^{2}$ Visually clear (homogeneous) gels are obtained if the water content during polymerization is less than about $40 \%$. Above this critical dilution, heterogeneous gels and eventually white spongy materials are formed. The heterogeneity above the critical dilution is caused by the poor compatibility of the polymer with water. Recently, employing the turbidity ratio method, Dusěk and Sedlácěk ${ }^{3}$ showed that PHEMA chains aggregate in visually clear gels.
Previously, the authors ${ }^{4}$ investigated the effect of water, which acts as a plasticizer or as an inert diluent during network formation, on the mechanical behavior of the networks. From a practical point of view, the mechanical behavior of PHEMA hydrogels with added salts is also interesting. A hydrogel contact lense should be boiled in water or in saline solution for the purpose of cleaning and sterilization. The swelling behavior of PHEMA hydrogels was extensively studied by Refojo. ${ }^{5}$ The swelling degree of PHEMA hydrogels depends markedly on the species of salts.

In this paper, it is our aim to investigate the effect of various added salts on the mechanical behavior and to compare the data for simple PHEMA hydrogels studied previously ${ }^{4}$ with the present data. 


\section{EXPERIMENTAL}

Samples

Homogeneous poly(2-hydroxyethyl methacrylate) hydrogel, sample W-40 from our previous study, ${ }^{4}$ was used. It was prepared by copolymerization of 2-hydroxyethyl methacrylate and a small amount of ethylene glycol dimethacrylate in the presence of $40 \%$ by volume of water. Pieces of water-equilibrated gel (W-40) were allowed to remain at room temperature in aqueous solutions $(0.1,0.3,0.6$, and $1 \mathrm{~mol} / \mathrm{l})$ of sodium chloride, sodium sulfate, and sodium iodide. After 3-months equilibrium swelling was assumed to be reached. We determined the water content in the standard manner, by blotting the superficial aqueous solution and weighing the specimens both in their swollen state and after drying to constant weight.

The weight fractions of water $\left(w_{1}\right)$ are shown in Figure 1. Gels equilibrated with sodium chloride solutions (series $\mathbf{A}$ ) and sodium sulfate solutions (series B) were used for dynamic measurements. Starting from gels equilibrated with $1 \mathrm{~mol} / l$ solution of sodium iodide, less swollen samples (series C) were also prepared in the following way: first deswollen by drying to the desired extent at room temperature, and then soaked in liquid paraffin (which is immiscible with the polymer) at $25^{\circ} \mathrm{C}$ for 1 day in order to insure homogeneous swelling throughout the sample. The $w_{1}$ 's are $0.160,0.180$, and 0.254 . Some characteristics of the samples used are given in Table I. $w_{\mathrm{s}}$ are the weight fractions of salt in the gels, in which the salt concentration was assumed to be that outside the

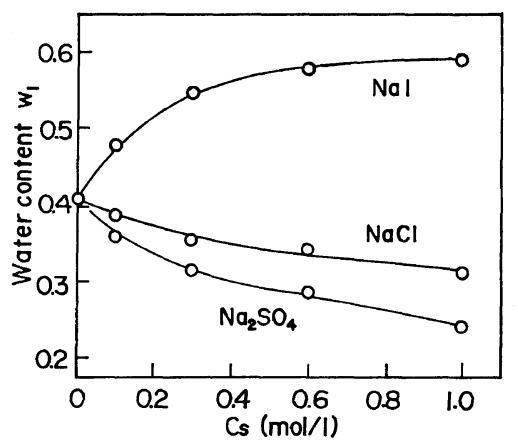

Figure 1. Effect of $\mathrm{NaCl}, \mathrm{Na}_{2} \mathrm{SO}_{4}$, and $\mathrm{NaI}$ on the swelling of PHEMA.
Table I. Some characteristics of the sample used

\begin{tabular}{lcccc}
\hline Sample & $w_{1}$ & $w_{\mathrm{s}}$ & $w_{\mathrm{s}}{ }^{\prime}$ & $v_{1, \mathrm{~s}}$ \\
\hline W-40 & 0.409 & & & \\
A-1 & 0.388 & 0.002 & & 0.453 \\
A-2 & 0.356 & 0.006 & & 0.422 \\
A-3 & 0.345 & 0.012 & & 0.414 \\
A-4 & 0.312 & 0.018 & 0.015 & 0.382 \\
B-1 & 0.361 & 0.005 & & 0.426 \\
B-2 & 0.315 & 0.013 & & 0.381 \\
B-3 & 0.288 & 0.025 & & 0.356 \\
B-4 & 0.242 & 0.035 & & 0.308 \\
C-1 & 0.254 & 0.167 & 0.171 & 0.399 \\
C-2 & 0.185 & 0.182 & & 0.322 \\
C-3 & 0.160 & 0.188 & & 0.293 \\
\hline
\end{tabular}

a W-40 from a previous study 4 : a copolymer of PHEMA with $0.5 \times 10^{-4} \mathrm{~mol} / \mathrm{cm}^{3}$ of ethylene glycol dimethacrylate prepared in the presence of $40 \%$ of water.

gels. The weight fractions of salt in the gels $w_{\mathrm{s}}{ }^{\prime}$ were determined for samples A-4 and C-1 from the elemental analysis. Results are also shown in Table I. $w_{\mathrm{s}}{ }^{\prime}$ is not equal to $w_{\mathrm{s}}$, because adsorption or desorption of salt with the polymer may occur. From the results of the elemental analysis we use $w_{\mathrm{s}}$ instead of $w_{\mathrm{s}}{ }^{\prime}$, taking account of the errors of about $20 \%$ with respect to the salt concentration of the gels. The volume fractions of salt solution $v_{1, \mathrm{~s}}$ were calculated using the equation $v_{1, \mathrm{~s}}=1 /\left[1+d_{2} w_{2} /\right.$ $\left.d_{1, \mathrm{~s}}\left(w_{1}+w_{\mathrm{s}}\right)\right] ; \quad d_{2}$ is the density of the polymer $\left(1.3 \mathrm{~g} / \mathrm{cm}^{3}\right)$ and $d_{1, \mathrm{~s}}$ that of salt solution.

Measurements of Complex Tensile Modulus

Measurements are carried out by use of a modified Fukuda and Date's apparatus ${ }^{6}$ in liquid paraffin in the frequency range 0.006 to $0.6 \mathrm{~Hz}$ and the temperature range 0 to $40^{\circ} \mathrm{C}$. The specimens were about $5 \mathrm{~cm}$ in length, $0.5 \mathrm{~cm}$ in width, and $0.1 \mathrm{~cm}$ in thickness. The measuring technique is described elsewhere. ${ }^{4}$

\section{RESULTS AND DISCUSSION}

\section{Transition Region}

The data for the sample B-4 are illustrated. Values of $E^{\prime}$ and $E^{\prime \prime}$ for this system are shown in Figures 2 and 3. Data for each system were superimposed to find $\log a_{T}$ values. The reduc- 


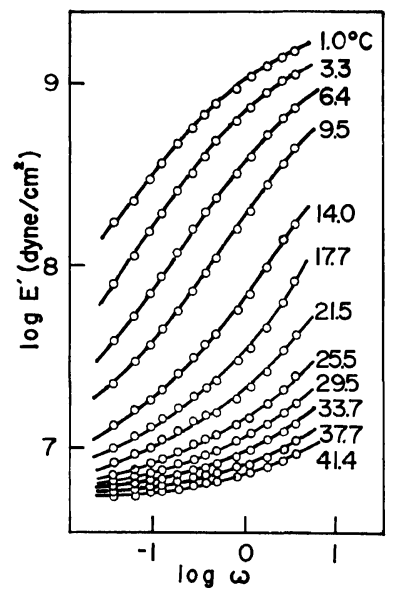

Figure 2. $\log E^{\prime}$ of B-4 plotted against $\log \omega$ at 12 temperatures as indicated.

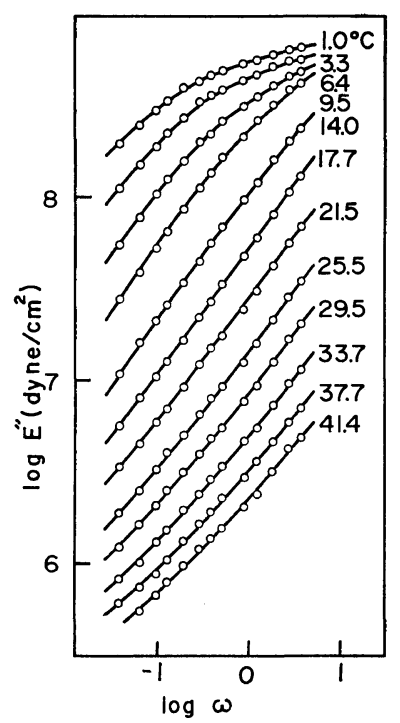

Figure 3. $\log E^{\prime \prime}$ of B-4 plotted against $\log \omega$ at 12 temperatures as indicated.

tion equations are: $E_{\mathrm{p}}{ }^{\prime}=E^{\prime}\left(T d / T_{0} d_{0}\right), \quad E_{\mathrm{p}}{ }^{\prime \prime}=$ $E^{\prime \prime}\left(T d / T_{0} d_{0}\right)$ where $d$ and $d_{0}$ are the densities at the absolute temperature $T$ of the measurements and the standard temperature $T_{0}$. Composite curves of $E_{\mathrm{p}}{ }^{\prime}$ and $E_{\mathrm{p}}{ }^{\prime \prime}$ for series $\mathrm{A}, \mathrm{B}$, and $\mathrm{C}$ are plotted logarithmically against the reduced circular frequency $\omega a_{T}$ in Figures 4 and 5. In these figures composite curves of the water-equilibrated sample $\mathrm{W}-40$, taken from ref 4 , are also shown.

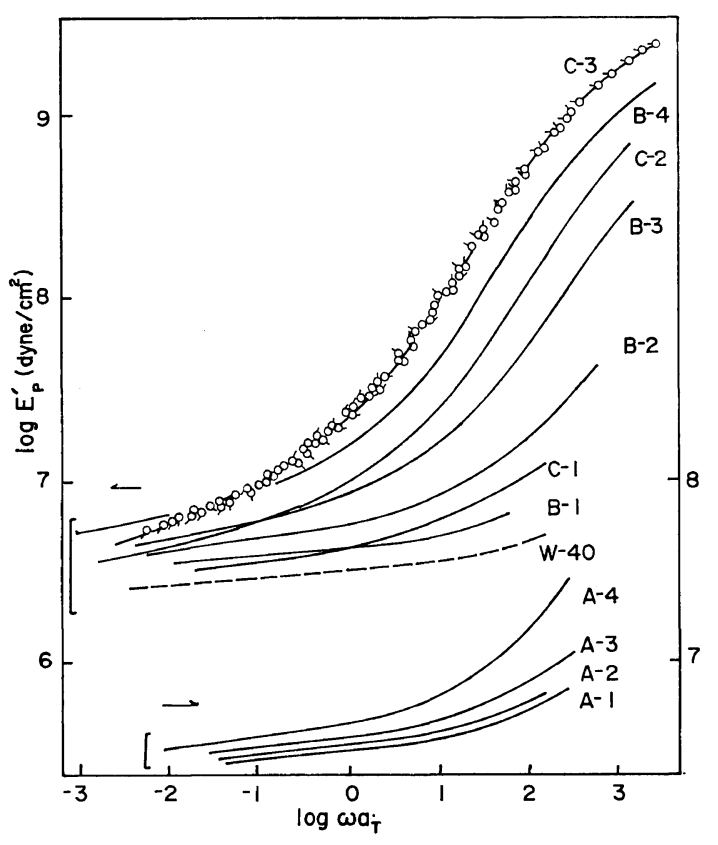

Figure 4. $\log E_{\mathrm{p}^{\prime}}$ vs. $\log \omega a_{T}$ for PHEMA hydrogels with added $\mathrm{NaCl}, \mathrm{Na}_{2} \mathrm{SO}_{4}$, and $\mathrm{NaI}$, at various temperatures reduced to $25^{\circ} \mathrm{C}$. Water and salt concentrations are listed in Table I. Measurements for $\mathrm{C}-3$ were performed at the temperatures, $1.4,7.9,13.2,18.0,24.5,29.6,34.4$, and $39.6^{\circ} \mathrm{C}$; pip pointing left denotes the lowest temperature and successive $45^{\circ}$ clockwise rotations denote successively higher temperatures. Similar sequences were used for the other systems. The curve of W-40 was taken from ref 4.

From the values of $\log a_{T}$, the constants $c_{1}$ and $c_{2}$ of the WLF equation ${ }^{7}$ were evaluated by the well-known procedure, for the reference temperature $25^{\circ} \mathrm{C}$. Results are shown in Table II.

The curves extending over 7 decades have the usual shape in the transition from rubber to glass-like consistency. Decreasing concentration of PHEMA shifts $\log E_{\mathrm{p}}{ }^{\prime}$ and $\log E_{\mathrm{p}}{ }^{\prime \prime}$ curves toward lower frequencies with relatively little change in shape at high-frequency. Viscoelastic properties in the transition region are supposed to be governed by short-range configurational motions of the polymer chain between coupling points on a molecule. ${ }^{7}$ The position of the transition zone reflects the magnitude of the monomeric friction coefficient $\zeta$. The storage and loss modulus curves at various concentra- 


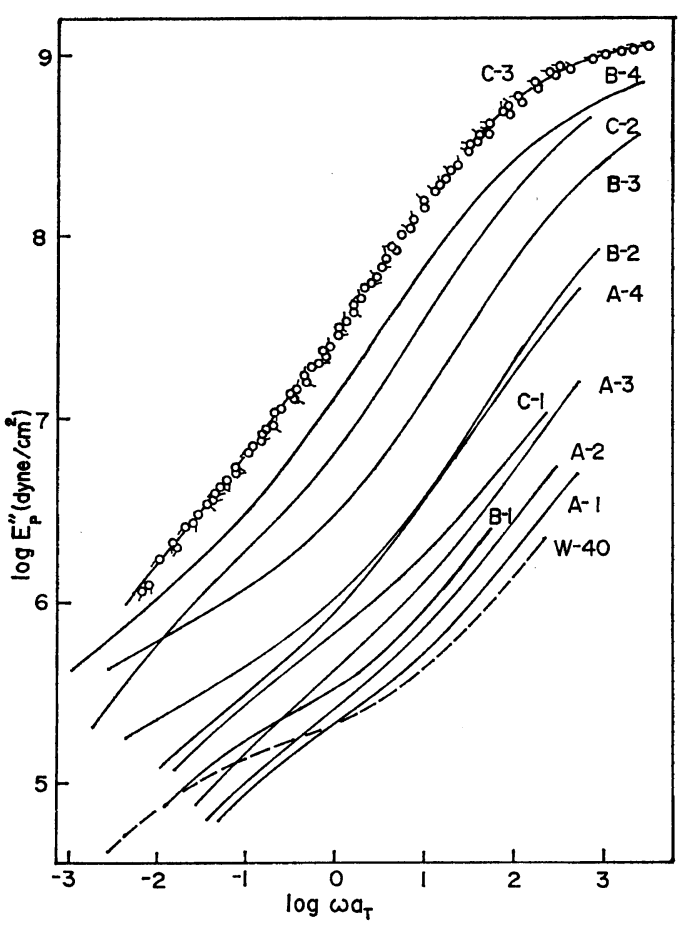

Figure 5. $\log E_{\mathrm{p}}{ }^{\prime \prime} v s . \log \omega a_{T}$ for PHEMA hydrogels with added $\mathrm{NaCl}, \mathrm{Na}_{2} \mathrm{SO}_{4}$, and $\mathrm{NaI}$. The data correspond to those in Figure 4.

Table II. $\log a_{c}$ and the constants of the WLF equation of various systems at $25^{\circ} \mathrm{C}$

\begin{tabular}{llll}
\hline Sample & $\log a_{c}$ & $c_{1}{ }^{0}$ & $c_{2}{ }^{0}, \operatorname{deg}$ \\
\hline W-40 & 0 & & \\
A-1 & 0.44 & & \\
A-2 & 0.61 & & \\
A-3 & 0.83 & 6.2 & 120 \\
A-4 & 1.43 & 9.3 & 130 \\
B-1 & 0.55 & & \\
B-2 & 1.60 & 8.6 & 115 \\
B-3 & 2.53 & 8.9 & 110 \\
B-4 & 3.51 & 9.1 & 96 \\
C-1 & 1.06 & & \\
C-2 & 3.04 & 12.3 & 135 \\
C-3 & 3.98 & 11.4 & 110 \\
\hline
\end{tabular}

tions should be reduced to a single curve in the transition region, if $\left(d_{2} / c\right) E^{\prime}(\omega)$ or $\left(d_{2} / c\right) E^{\prime \prime}(\omega)$ is plotted against $\omega a_{c}{ }^{\prime}$. Here $d_{2}$ is the density of pure polymer, $c$ is the polymer concentration in grams per $\mathrm{cm}^{3}$, and $a_{c}^{\prime}$ is the shift factor on the frequency scale, which can be regarded as the ratio of $\zeta$ in solution to $\zeta$ in undiluted polymer. The reduced variables may be rewritten in terms of a reference concentration $c_{0}$ other than the undiluted polymer in the form, $E_{c}{ }^{\prime}(\omega)=\left(c_{0} / c\right) E_{c_{0}}^{\prime}\left(\omega a_{c}\right)$ and $E_{c}^{\prime \prime}(\omega)=\left(c_{0} / c\right) E_{c_{0}}^{\prime \prime}\left(\omega a_{c}\right)$ where $a_{c}$ is defined as the ratio $a_{c}{ }^{\prime}(c) / a_{c}{ }^{\prime}\left(c_{0}\right)$. In a present study, $0.618 \mathrm{~g} / \mathrm{cm}^{3}$ of the water-equilibrated sample W-40, which was studied previously, ${ }^{4}$ was chosen as the reference $c_{0}$. The values of $a_{c}$ are given in Table II.

Free Volume Parameters. In a previous paper, ${ }^{4}$ values of $\zeta$ for W-40 swollen with water (the volume fraction of water $v_{1}=0.475,0.434,0.418$, 0.378 , and 0.326 ) were calculated with the aid of ${ }^{7}$

$$
\begin{aligned}
\log \zeta= & 2 \log H+\log \tau+\log (6 / k T) \\
& +2 \log \left(2 \pi M_{0} / a c N_{0}\right)
\end{aligned}
$$

from the part of the spectrum $H$ where the logarithmic dependence has the theoretically predicted slope $-1 / 2$. In eq $1, k$ denotes the Boltzmann constant, $N_{0}$ is Avogadro's number, and $a^{2}$ is the mean-square molecular length per monomer unit. Here $a$ was taken as $7 \times 10^{-8} \mathrm{~cm} .^{8}$

The friction coefficient $\log \zeta_{1, \mathrm{~s}}$ for the systems with added salt, defined as $\log \zeta_{1, \mathrm{~s}}=\log a_{c}+$ $\log \zeta_{\mathrm{W}-40}$, is given in Table III. Here $\log a_{c}$ is the shift factor obtained by the method of the time-concentration superposition (Table II), and $\log \zeta_{\mathrm{W}-40}$ for the water-equilibrated sample W-40 is $-3.84 \mathrm{dyn} \mathrm{sec} / \mathrm{cm}$.

Table III. Free volume parameters and the $\zeta$ values for PHEMA hydrogels with added salts at $25^{\circ} \mathrm{C}$

\begin{tabular}{lrrrrrr}
\hline & & & \multicolumn{3}{c}{$f\left(v_{1, \mathrm{~s}}\right)$} \\
\cline { 5 - 7 } Sample & $\log \zeta_{1, \mathrm{~s}}$ & $\log \zeta_{w}$ & $\log \zeta_{0}$ & (a) & (b) & (c) \\
\hline W-40 & $-3.84^{\mathrm{a}}$ & & & $0.101^{\mathrm{a}}$ & $0.058^{\mathrm{a}}$ \\
A-1 & -3.40 & -3.60 & -3.60 & 0.092 & 0.055 & \\
A-2 & -3.23 & -3.26 & -3.25 & 0.089 & 0.054 & \\
A-3 & -3.06 & -3.17 & -3.13 & 0.086 & 0.053 & 0.070 \\
A-4 & -2.41 & -2.74 & -2.68 & 0.076 & 0.049 & 0.047 \\
B-1 & -3.29 & -3.31 & -3.29 & 0.090 & 0.055 & \\
B-2 & -2.24 & -2.73 & -2.71 & 0.074 & 0.048 & 0.049 \\
B-3 & -1.31 & -2.36 & -2.31 & 0.063 & 0.044 & 0.048 \\
B-4 & -0.33 & -1.46 & -1.35 & 0.056 & 0.040 & 0.048 \\
C-1 & -2.78 & -2.99 & -2.47 & 0.082 & 0.047 & \\
C-2 & -0.80 & -1.74 & -0.64 & 0.059 & 0.042 & 0.035 \\
C-3 & 0.14 & -1.11 & 0.38 & 0.053 & 0.038 & 0.038 \\
\hline
\end{tabular}

a Values taken from ref 4. 
Previously, ${ }^{4}$ from the dependence of $\zeta$ on the volume fraction of water $v_{1}$, the free volume parameters were calculated by use of two types of the free volume equation. One is the FujitaKishimoto equation in the form ${ }^{9,10}$

$$
\frac{-\left(v_{1}-v_{1}^{0}\right)}{\log a_{c}}=2.303 \frac{f\left(v_{1}^{0}\right)^{2}}{\beta}+2.303 f\left(v_{1}^{0}\right)\left(v_{1}-v_{1}^{0}\right)
$$

Here, $a_{c}$ is the ratio between the friction coefficients $\zeta$ at the concentrations $v_{1}$ and $v_{1}{ }^{0}$, and $\beta$ characterizes the concentration dependence of the fractional free volume. The other is the Berry-Fox equation ${ }^{11}$ recast by the authors into the form ${ }^{4}$

$$
\begin{aligned}
\frac{-\left(v_{1}-v_{1}^{0}\right)}{\log a_{c}}= & \frac{2.303 f\left(v_{1}^{0}\right)^{2}}{\alpha_{\mathrm{f}}\left(v_{1}^{0}\right) T_{\mathrm{g}}\left(v_{1}^{0}\right)^{2} k^{\prime}}+2.303 f\left(v_{1}^{0}\right) \\
& \times\left[\frac{f\left(v_{1}^{0}\right)}{\alpha_{\mathrm{f}}\left(v_{1}^{0}\right) T_{\mathrm{g}}\left(v_{1}^{0}\right)}+1\right]\left(v_{1}-v_{1}^{0}\right)
\end{aligned}
$$

Here, $\alpha_{\mathrm{f}}$ is the thermal expansion coefficient of the fractional free volume, $T_{\mathrm{g}}\left(v_{1}{ }^{0}\right)$ is the glass transition temperature at concentration $v_{1}{ }^{0}$, and $k^{\prime}$ is a constant, defined as

$$
k^{\prime}=\frac{1}{v_{1}}\left[\frac{1}{T_{\mathrm{g}}\left(v_{1}\right)}-\frac{1}{T_{\mathrm{g}}(0)}\right]
$$

The $-\left(v_{1}-v_{1}^{0}\right) / \log a_{c} \quad v s . \quad\left(v_{1}-v_{1}^{0}\right)$ plot was made to test their applicability to experimental data for PHEMA hydrogels. The values of $f\left(v_{1}{ }^{0}=0.326\right)$ and $\beta$ estimated from eq 2 are 0.069 and 0.217 at $25^{\circ} \mathrm{C}$, respectively. The values of $T_{\mathrm{g}}\left(v_{1}{ }^{0}=0.326\right)$ and $k^{\prime}$ obtained from a dilatometric measurement are $248^{\circ} \mathrm{K}$ and $4.0 \times$ $10^{-3} \mathrm{deg}^{-1}$, respectively. Using these values, the $f\left(v_{1}^{0}=0.326\right)$ and $\alpha_{\mathrm{f}}\left(v_{1}^{0}=0.326\right)$ values estimated from eq 3 are 0.046 and $3.8 \times 10^{-4} \mathrm{deg}^{-1}$, respectively. According to the Fujita-Kishimoto theory and the modified Berry-Fox theory, the dependence of $f$ on $v_{1}$ is expressed by the equations

$$
\begin{gathered}
f\left(v_{1}\right)=f\left(v_{1}{ }^{0}\right)+\beta\left(v_{1}-v_{1}{ }^{0}\right) \\
f\left(v_{1}\right)=f\left(v_{1}{ }^{0}\right)+\frac{\alpha_{\mathrm{f}}\left(v_{1}{ }^{0}\right) T_{\mathrm{g}}\left(v_{1}\right)^{2} k^{\prime}\left(v_{1}-v_{1}{ }^{0}\right)}{1+k^{\prime} T_{\mathrm{g}}\left(v_{1}^{0}\right)\left(v_{1}-v_{1}^{0}\right)}
\end{gathered}
$$

The water concentration $v_{1}{ }^{\mathrm{g}}$ corresponding to $T_{\mathrm{g}}=25^{\circ} \mathrm{C}$ is $0.157 .^{4}$ Choosing 0.157 as a reference concentration, we can rewrite eq 2 in the following equation:

$$
\log \zeta=\log \zeta_{\mathrm{g}}-\frac{1}{2.303 f_{\mathrm{g}}}\left[\frac{\beta\left(v_{1}-v_{1}^{\mathrm{g}}\right)}{f_{\mathrm{g}}+\beta\left(v_{1}-v_{1}^{\mathrm{g}}\right)}\right]
$$

where the fractional free volume $f_{\mathrm{g}}$ at $T_{\mathrm{g}}\left(v_{1}{ }^{\mathrm{g}}\right)$ is 0.032 by use of eq 5 , the monomeric friction coefficient $\log \zeta_{\mathrm{g}}$ at $T_{\mathrm{g}}\left(v_{1}{ }^{\mathrm{g}}\right)$ is $5.43 \mathrm{dyn} \mathrm{sec} / \mathrm{cm}$, and $\beta$ is 0.217 . $\log \zeta$ changes linearly with $\beta\left(v_{1}-v_{1}^{\mathrm{g}}\right) /$ $\left[f_{\mathbf{g}}+\beta\left(v_{1}-v_{1}{ }^{\mathrm{g}}\right)\right]$. Assuming that the values of $f_{\mathrm{g}}$ and $\log \zeta_{\mathrm{g}}$ for the systems with added salts are equal to those for the system without added salts, $f\left(v_{1, \mathrm{~s}}\right)\left[=f_{\mathrm{g}}+\beta\left(v_{1, \mathrm{~s}}-v_{1, \mathrm{~s}}^{\mathrm{g}}\right)\right]$ values can be determined by use of the plot of $\log \zeta$ versus the term of eq 7 in the bracket; the value of the abscissa corresponding to $\log \zeta=\log \zeta_{1, \mathrm{~s}}$ (Table III) is equal to $1-\left[f_{\mathrm{g}} / f\left(v_{1, \mathrm{~s}}\right)\right] . \quad f\left(v_{1, \mathrm{~s}}\right)$ values are given in column (a) of Table III.

Similarly, eq 3 is rewritten in the following equation

$$
\begin{aligned}
\log \zeta= & \log \zeta_{\mathrm{g}}-\frac{1}{2.303 f_{\mathrm{g}}} \\
& \times\left[\frac{\frac{\alpha_{\mathrm{f}}\left(v_{1}^{\mathrm{g}}\right) T_{\mathrm{g}}\left(v_{1}^{\mathrm{g}}\right)^{2} k^{\prime}\left(v_{1}-v_{1}^{\mathrm{g}}\right)}{1+k^{\prime} T_{\mathrm{g}}\left(v_{1}^{\mathrm{g}}\right)\left(v_{1}-v_{1}^{\mathrm{g}}\right)}}{f_{\mathrm{g}}+\frac{\alpha_{\mathrm{f}}\left(v_{1}^{\mathrm{g}}\right) T_{\mathrm{g}}\left(v_{1}^{\mathrm{g}}\right)^{2} k^{\prime}\left(v_{1}-v_{1}^{\mathrm{g}}\right)}{1+k^{\prime} T_{\mathrm{g}}\left(v_{1}^{\mathrm{g}}\right)\left(v_{1}-v_{1}^{\mathrm{g}}\right)}}\right]
\end{aligned}
$$

where $f_{\mathrm{g}}$ is 0.027 by use of eq $\left.6, \alpha_{\mathrm{f}} T_{\mathrm{g}}\left(v_{1}\right)^{\mathrm{g}}\right)^{2} k^{\prime}$ is 0.135 , and $k^{\prime} T_{\mathrm{g}}\left(v_{1}^{\mathrm{g}}\right)$ is 1.19 . The value of the abscissa corresponding to $\log \zeta=\log \zeta_{1, \mathrm{~s}}$ is also equal to $1-\left[f_{\mathrm{g}} / f\left(v_{1, \mathrm{~s}}\right)\right]$ in the plot of $\log \zeta$ versus the term of eq 8 in the bracket. Results are shown in column (b) of Table III. $f\left(v_{1, \mathrm{~s}}\right)$ values estimated from the equation, $f\left(v_{1, \mathrm{~s}}\right)=$ $1 / 2.303 c_{1}{ }^{0}$, are given in column (c) of Table III. Previously ${ }^{4}$ it was found that the $f\left(v_{1}{ }^{0}=0.326\right)$ value derived from the analysis of the dependence of $\log a_{T}$ on $T$ is 0.043 , and this value is in close agreement with the $f\left(v_{1}{ }^{0}=0.326\right)$ value estimated from the modified Berry-Fox equation. From Table III, it follows that the agreement between the $f\left(v_{1, \mathrm{~s}}\right)$ values calculated from $c_{1}$ parameters and those from eq 8 is satisfactory. This again supports the previous statement ${ }^{4}$ that the dependence of $\zeta$ on $v_{1}$ for the homogeneous hydrogels (W-40) is described by the concept of the free volume on the basis of the modified Berry-Fox equation.

Effects of the Addition of Salts on the Monomeric Friction Coefficient. In order to compare the effect of the addition of salts on $\zeta$, we estimated the friction coefficient $\log \zeta_{w}$ at the 


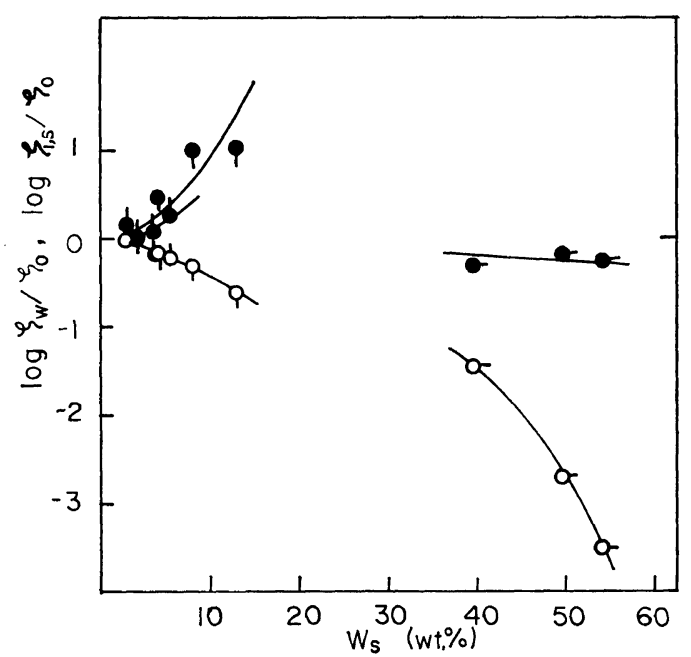

Figure 6. $\log \zeta_{1, \mathrm{~s}} / \zeta_{0}$ and $\log \zeta_{w} / \zeta_{0}$ plotted against $W_{\text {s. }}$ Pips pointing up, down, and right denote series $\mathrm{A}, \mathrm{B}$, and $\mathrm{C}$, respectively. Filled circles denote $\log \zeta_{1, \mathrm{~s}} / \zeta_{0}$, and open ones $\log \zeta_{w} / \zeta_{0}$.

concentration $v_{1}$ equal to the volume fraction of aqueous salt solution $v_{1, \mathrm{~s}}$, and the friction coefficient $\log \zeta_{0}$ at the concentration $v_{1}$ corresponding to $w_{\mathrm{s}}$ (Table $\mathrm{I}$ ) $=0$ from eq $8 . \quad \log \zeta_{1, \mathrm{~s}} /$ $\zeta_{0}$ and $\log \zeta_{w} / \zeta_{0}$ are plotted as functions of the weight percent of salts $w_{\mathrm{s}}\left[=100 w_{\mathrm{s}} /\left(w_{1}+w_{\mathrm{s}}\right)\right]$ in Figure 6. From Figure 6, it follows that $\log \zeta_{1, \mathrm{~s}} /$ $\zeta_{0}$ for the gels with added sodium chloride and sodium sulfate has a positive value and increases with increasing $W_{\mathrm{s}}$, and that, for the gels with added sodium iodide, $\log \zeta_{1, \mathrm{~s}} / \zeta_{0}$, on the contrary, has a slightly negative value. The addition of sodium chloride and sodium sulfate causes the increase in $\zeta$, and sodium iodide causes the reduction of $\zeta$ in the gel. As was remarked in the experimental section, the exact value of salt concentration 'in the gel' cannot be estimated. However, the above-mentioned results are not much affected by the uncertainty with respect to $w_{\mathrm{s}}$. For example, $\log \zeta_{0}$ values calculated from eq 8 by use of the analytical concentration for A-4 and $\mathrm{C}-1$ are -2.67 and -2.49 respectively. These values are very similar to $\log \zeta_{0}$ in Table III. This peculiar plasticizing effect observed for sodium iodide, though it is much less than that of water ( $c f$. $\log \zeta_{1, \mathrm{~s}}$ and $\left.\log \zeta_{w}\right)$, will be discussed later.

In a previous paper, ${ }^{12}$ we reported that the effect of plasticizers on $\zeta$ depends on their own rheological properties, i.e., their viscosity or $T_{\mathrm{g}}$. The viscosity of aqueous solutions of sodium chloride and sodium sulfate increases with increasing salt concentration. Accordingly, the addition of sodium chloride and sulfate causes a decrease in the plasticizing effect of water, in other words, a reduction of free volume of water. It seems that this effect is responsible for the increase in $\zeta$ for the gels with added sodium chloride and sodium sulfate. Sodium iodide also has the effect of increasing the water viscosity, which is comparable to sodium sulfate in the concentration range tested. ${ }^{13}$ This suggests that it is necessary to take account of the influence of sodium iodide not only on water but also on PHEMA. In analogy with the work of Lundberg, et al., ${ }^{14}$ on the interaction of inorganic salts with poly(ethylene oxide), it seems quite conceivable that the iodide anion is the species directly associating with the polymer. In this connection, Refojo ${ }^{5}$ reported in a study of swelling behavior that the mechanism by which ion-polymer interaction occurs is not clear, but that in the case of the iodide ion it is very likely to be ion-dipole interactions. Thus we believe that the plasticizing effect observed for the gels with added sodium iodide reflects the specific interaction of the iodide ion with PHEMA.

\section{Rubbery Region}

Time-Concentration Superposition. For a better comparison of the low-frequency area, we con-

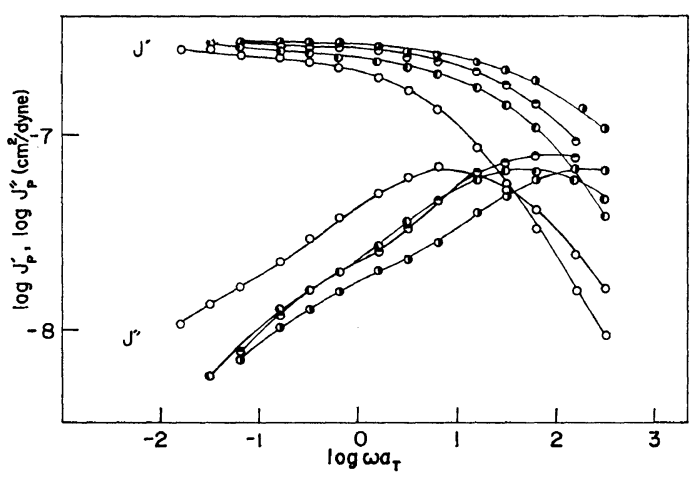

Figure 7. $\log J_{\mathrm{p}^{\prime}}$ and $\log J_{\mathrm{p}}{ }^{\prime \prime}$ versus $\log \omega a_{T}$ for PHEMA with added NaCl: (O), A-1; (Ө), A-2; (D), A-3; (O), A-4. 


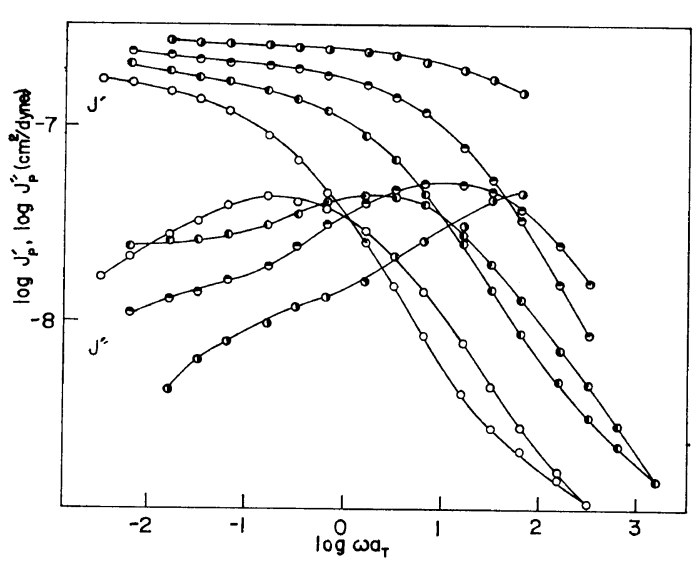

Figure 8. $\log {J_{\mathrm{p}}}^{\prime}$ and $\log J_{\mathrm{p}^{\prime \prime}}{ }^{\prime \prime}$ versus $\log \omega a_{T}$ for PHEMA with added $\mathrm{Na}_{2} \mathrm{SO}_{4}$ : ((), B-1; (Ө), B2; (O), B-3; (○), B-4.

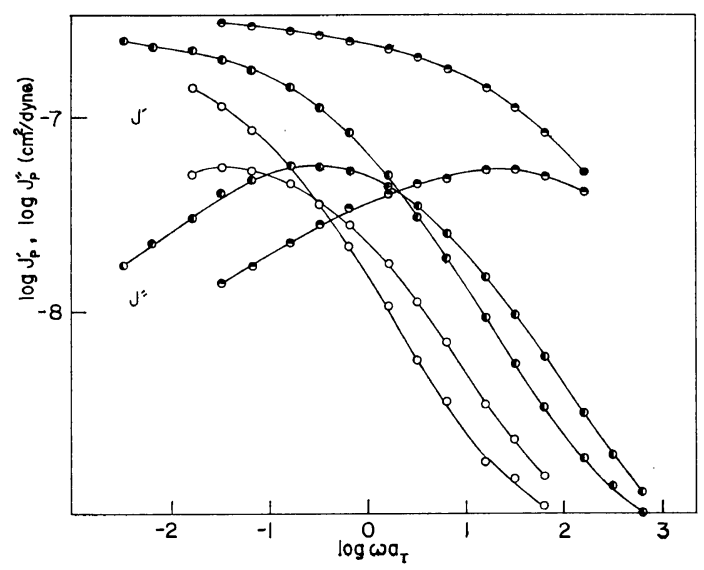

Figure 9. $\log J_{p^{\prime}}$ and $\log J_{p^{\prime}}{ }^{\prime \prime}$ versus $\log \omega a_{T}$ for PHEMA with added NaI: (Ө), C-1; (D), C-2; (O), C-3.

verted the $\log E_{\mathrm{p}}{ }^{\prime}$ and $\log E_{\mathrm{p}}{ }^{\prime \prime}$ values from Figures 4 and 5 to $J^{\prime}$ and $J^{\prime \prime}$ using $\log E_{\mathrm{p}}{ }^{\prime}=$ $-\log J_{\mathrm{p}}{ }^{\prime}-\log \left(1+\tan ^{2} \delta\right) . \quad \log J_{\mathrm{p}}{ }^{\prime}$ and $\log J_{\mathrm{p}}{ }^{\prime \prime}$ for series $\mathrm{A}, \mathrm{B}$, and $\mathrm{C}$ are plotted against $\log \omega a_{T}$ in Figures 7-9.

In order to compare the effect of the addition of salts on the shapes of $J^{\prime}$ and $J^{\prime \prime}, J_{c}^{\prime}=J_{c_{0}}^{\prime}\left(c / c_{0}\right)$ and $J_{c}{ }^{\prime \prime}=J_{c_{0}}^{\prime \prime}\left(c / c_{0}\right)$ for samples A-4, B-2, and $\mathrm{C}-1$ can be superposed by utilizing $\log a_{c}$ previously obtained with $\mathrm{W}-40$ swollen to $v_{1}$ of 0.382 as a reference state; results are shown in Figure 10. The polymer volume fractions of $\mathrm{A}-4, \mathrm{~B}-2$, and $\mathrm{C}-1$ are very similar to that of W-40. Composite curves of B-4, C-2, and
W-40 swollen to $v_{1}$ of 0.326 are also plotted against $\log \omega a_{c}$ in Figure 11. In Figures 10 and 11 the time-concentration superposition was successful in the transition region, as seen in Figures 4 and 5. But in the rubber-like region $J_{c}^{\prime}$ takes higher values with the addition of sodium iodide, and lower values with the addition of sodium chloride and sodium sulfate, than in the simple PHEMA gels. Therefore, the concentration reduction which is used in the superposition of the main transition region cannot be applied to the rubbery region of the systems with added salts. It was found previously ${ }^{4}$ that for simple PHEMA hydrogels the

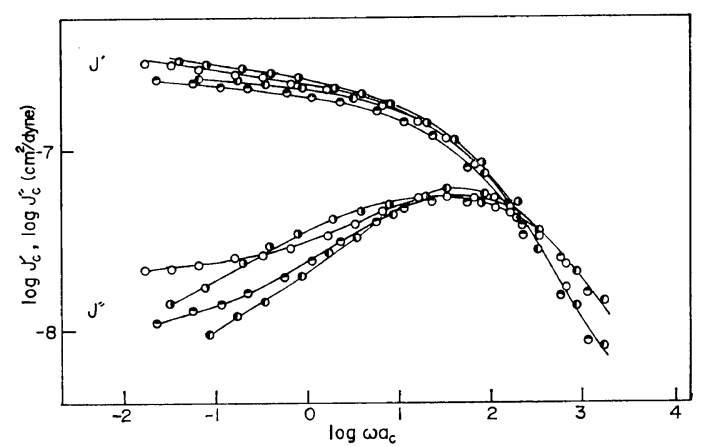

Figure 10. Composite curve for A-4, B-2, C-1, and $\mathrm{W}-40$ swollen to $v_{1}$ of 0.382 plotted against

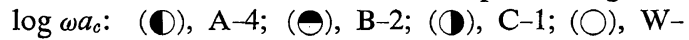
40. Compliances and the circular frequency were reduced to those of $\mathrm{W}-40$ swollen to $v_{1}$ of 0.382 (ref 4).

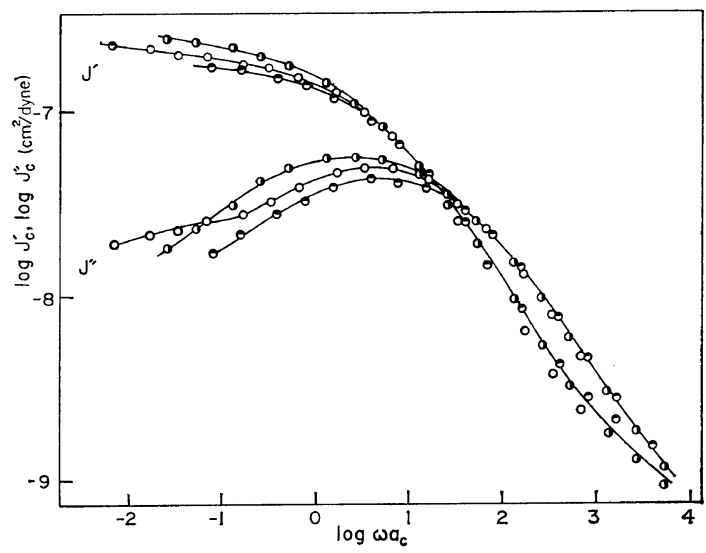

Figure 11. Composite curve for B-4, C-2, and W-40 swollen to $v_{1}$ of 0.326 plotted against $\log \omega a_{c}$ : (Ө), B-4; (C), C-2; (○), W-40. Compliances and the circlar frequency were reduced to those of W-40 swollen to $v_{1}$ of 0.326 (ref 4). 


\section{K. NAKAMURA}

time-concentration superposition is successful in both the transition and rubbery regions.

For the dependence of compliance on the degree of swelling $v_{2}$, we also have, from the kinetic theory of the rubber elasticity, ${ }^{15}$ the relationship $^{16} \quad J_{v_{2}}^{\prime 0}=J_{v_{2}}^{\prime}\left(v_{2} / v_{2}{ }^{0}\right)^{1 / 3}$. However this correction factor is negligible compared to the present experimental uncertainty, because the systems in Figures 10 and 11 are very similar to each other with respect to the polymer concentration $v_{2}$. These results suggest that a deviation from the superposition caused by the addition of salts corresponds to a change in some structure of the PHEMA hydrogels.

An additional reduction deduced by analogy with the so-called $f$-shift ${ }^{7}$ was made to test its applicability to the data in Figures 10 and 11: $\log J_{c}{ }^{\prime} f^{\prime}$ or $\log J_{c}{ }^{\prime \prime} f^{\prime}$ is plotted against $\log \omega a_{c} / f^{\prime 2}$. Here the degree of polymerization $P_{c}$ changes by a factor $1 / f^{\prime}$. It was found that this reduction is successful in the rubbery region, except for a slight deviation in the region of the maximum in $J^{\prime \prime}$ for A-4. With PHEMA hydrogels without added salts as a reference state, $\log f^{\prime}$ values of $\mathrm{C}-2, \mathrm{C}-1, \mathrm{~B}-4, \mathrm{~B}-2$, and $\mathrm{A}-4$ are $-0.09_{6},-0.05_{0}, 0.05_{6}, 0.02_{4}$, and $0.04_{8}$, respectively. Accordingly, a deviation from the reduction used in the superposition of the main transition region caused by the addition of salts is supposed to be attributable to a change in $P_{c}$.

Dusěk and Sedlácěk ${ }^{3}$ reported from optical measurements that PHEMA gels generally regarded as homogeneous contain heterogeneities of several microns in diameter. These heterogeneities are most probably formed during polymerization; in the swollen state they manifest themselves by local differences in the concentration of the polymer. A similar phenomenon was observed by Prins, et al., in measuring the light scattering ${ }^{17}$ and stress-induced birefrigence $^{18,19}$ of gels. They suggested that these heterogeneities should not be considered as rigid rods in an amorphous matrix, and that diluentinduced aggregation phenomena could be expected, possibly leading to some local mesomorphic order of either nematic or smectic type for PHEMA gels. Presumably the existence of this diluent-induced structure causes the reduction of the compliances of gels, leading to an increase in the number of coupling points. How- ever, the behavior of the friction coefficient in the transition region may not be much affected by the existence of such structure, because the viscoelastic properties in the transition region are supposed to be attributable to the motions of a short segment of the polymer, which are oblivious of the number of coupling points. ${ }^{7}$

It was observed by Sedlácěk, et al., ${ }^{20}$ that samples prepared in the presence of diluent were perfectly transparent after polymerization, but turned optically heterogeneous if the solvent was replaced by a poorer one. Chlorides and sulfates decrease the solubility of PHEMA in water (salting-out), and iodides excercise saltingin on PHEMA, as shown in Figure 1. These tendencies are in favor of the position of these ions in the lyotropic series. We can regard aqueous solutions of sodium chloride and sodium sulfate as poorer solvents than water, and those of sodium iodide as better ones. Accordingly the decrease of the reduced compliance $J_{c}^{\prime}$ for the systems with added sodium chloride and sodium sulfate is attributed to an increase in diluent-induced structure with the addition of these salts. For the systems with added sodium iodide, a reinforcement effect caused by a diluent-induced structure decreases with the addition of sodium iodide. Thus, the effect of the addition of salts on the reduced compliance is well interpreted in terms of the difference in the influence of the added salts on a diluentinduced structure in PHEMA hydrogels.

Acknowledgement. The author wishes to express his sincere thanks to Prof. Tsurutaro Nakagawa and Dr. Tsuyoshi Komatsu for their guidance and helpful discussions during the course of the present study. The present work was supported in part by a Grant for Scientific Research from the Ministry of Education.

\section{REFERENCES}

1. O. Wichterle and D. Lim, Nature (London), 185, 117 (1960).

2. J. Janácěk, J. Macromol. Sci.-Rev. Macromol. Chem., C(9), 1 (1973).

3. K. Dusěk and B. Sedlácěk, Collect. Czech. Chem. Commun., 34, 136 (1969).

4. K. Nakamura and T. Nakagawa, J. Polym. Sci., Part $A-2$, in press. 
5. M. F. Refojo, ibid., Part A-1, 5, 3103 (1967).

6. F. Fukuda and M. Date, Japan. J. Appl. Phys., 1, 59 (1962).

7. J. D. Ferry, "Viscoelastic Properties of Polymers," John Wiley, New York, N.Y., 1961.

8. M. Ilavský, J. Hasa, and J. Janácěk, Collect. Czech. Chem. Commun., 33, 3197 (1968).

9. H. Fujita and A. Kishimoto, J. Polym. Sci., 28, 547 (1958).

10. H. Fujita, A. Kishimoto, and K. Matsumoto, Trans. Faraday Soc., 56, 424 (1960).

11. G. C. Berry and T. G Fox, Advan. Polym. Sci., 5, 261 (1968).

12. K. Nakamura and T. Nakagawa, Nippon Kagaku Zasshi (J. Chem. Soc. Japan, Pure Chem. Sect.), 89, 38 (1968).
13. I.C.T., V, p. 15 (1929).

14. R. D. Lundberg, F. E. Bailey, and R.W. Callard, J. Polym. Sci., Part A-1, 4, 1563 (1966).

15. L. R. G. Treloar, "The Physics of Rubber Elasticity," University of Oxford Press, Oxford, 1958.

16. M. Ilavský and J. Hasa, Collect. Czech. Chem. Commun., 34, 2199 (1969).

17. J. H. Gouda, K. Povodater, T. C. Warren, and W. Prins, Polym. Lett., 8, 225 (1970).

18. M. Ilavský and W. Prins, Macromolecules, 3, 415 (1970).

19. M. Ilavský and W. Prins, ibid., 3, 425 (1970).

20. B. Sedlácěk, Collect. Czech. Chem. Commun., 32, 1398 (1967). 